\title{
FACTEURS LIES AU RENDEMENT DANS LA RAFFINERIE DE CANNE DU PERIMETRE SUCRIER DE FERKE 1 EN CÔTE D'IVOIRE
}

\author{
F.K.TOURE' ${ }^{1}$ BOHOUSSOU' ${ }^{1}$, E. KOFFI' et G. N. AGBO'1 \\ ${ }^{1}$ Université de Cocody, UFR Biosciences, LABSA, BP 42 Abidjan 22. \\ E. mail : fabien_toure@yahoo.fr
}

\begin{abstract}
RESUME
L'efficacité de la raffinerie de canne de SUCAF- CI (Ferké 1) à Ferkessédougou, au nord de la Côte d'Ivoire, a été évaluée à partir des facteurs qui influent sur le rendement usine au cours des campagnes sucrières de 2003, 2004, 2005 et 2006. La production du sucre blanc raffiné a été effectué, par broyage de tiges de cannes issues de 11 variétés de début, de milieu et de fin de campagne, puis par extraction et concentration du sucre obtenu. L'extraction réelle, le rendement et la richesse de l'usine, ainsi que le gain de pureté pendant l'épuration, la chute de pureté pendant l'évaporation et l'épuisement de la masse cuite $\mathrm{C}$ ont été, en moyenne, respectivement de 90,$6 ; 9,6 ; 12,9 ; 1,0 \% ; 0,8$ point et $23,6 \%$. Le rendement de l'usine, avec peu de variations, a principalement été affecté par la richesse de la canne et la chute de la pureté du jus pendant l'évaporation. L'amélioration du rendement de l'usine de Ferké 1 et de la qualité du sucre passent par la réduction du délai entre la coupe et le broyage des tiges de canne.
\end{abstract}

Mots clés : Canne à sucre, raffinerie, rendement, processus de production, richesse usine.

\section{ABSTRACT}

FACTORS AFFECTING THE YIELD OF THE FERKE 1 SUGARCANE REFINERY IN CÔTE D'IVOIRE

The efficiency of the cane refinery of SUCAF- CI (Ferké 1) in Ferkessédougou, in the north of Côte d'Ivoire, was evaluated using the factors affecting factory yield during the sugarcane harvest campaigns of 2003, 2004, 2005 and 2006. Refined white sugar was produced by milling canes from 11 beginning middle and end campaigns varieties of sugar cane to extract and the juice concentrate. The means of the actual extraction, yield and sugar content of the factory, purity gain during purification, purity drop during the evaporation process and the using up of the cooked mass $C$ were respectively of $90.6,9.6,12.9,1.0 \%, 0.8$ point and $23.6 \%$. Factory yield, with low variation, was affected mainly by sugar content and, purity drop during the evaporation process. Increasing the improvement of yield at the Ferké 1 sugar refinery yield and product quality require a substantial reduction in the delay between the talk cutting and the milling step.

Key words : Cane sugar, refinery, yield, process of production, sugar content. 


\section{INTRODUCTION}

La production sucrière ivoirienne, qui couvre la demande intérieure, varie entre 150000 et 180000 t par an. Elle est assurée par deux opérateurs privés repreneurs de l'ex-société d'état Sodesucre (société pour le développement de la canne à sucre) depuis juin 1997 (Pene et Kehe, 2005).

Pour la campagne 1998 - 1999, la production sucrière ivoirienne a été repartie de la manière suivante : $79200 \mathrm{t}$ de sucre roux dont $1300 \mathrm{t}$ de sucre en morceaux, $59655 \mathrm{t}$ de sucre blanc et industriel, dont 77000 t de sucre en morceaux. La consommation ivoirienne en sucre est estimée à 162000 t en 1999, avec une croissance d'environ 4,9\% par an sur la période de 1996 à 1999. Le sucre roux occupe une place prépondérante, avec $53 \%$ des volumes vendus, suivi par le sucre blanc en morceaux $(20 \%)$ et le sucre blanc cristallisé $(1,5 \%)$ et la filière agroalimentaire absorbe $12 \%$ des volumes sous forme de sucre blanc industriel.

L'Union Economique et Monétaire de l'Afrique de l'Ouest (UEMOA) représente un marché potentiel de 63 millions de consommateurs. Au sein des 8 pays, la production sucrière est environ 330000 t et ne couvre que $50 \%$ de la consommation. La production sucrière ivoirienne représente $54 \%$ de la production des états Ouest-africains et $26 \%$ de la demande. Ce marché offre, à moyen terme, les perspectives de développement des entreprises ivoiriennes SUCAF-CI (Sucrerie d'Afrique de Côte d'Ivoire) et SUCRIVOIRE (Sucre de Côte d'Ivoire), http:/ /www.bnetd.cil, 2010.

Pour couvrir cette demande intérieure et honorer les engagements internationaux, notamment les quotas USA, ACP (Afrique, Caraïbes, Pacifique) etc., les sucreries à canne ivoiriennes n'ont d'alternative que la maîtrise du processus de fabrication du sucre, puisque les surfaces plantées sont déjà fixées. La couverture de la demande, sans cesse croissante, peut en effet être possible avec une amélioration de la productivité des différents paramètres du processus de fabrication du sucre. C'est notamment le cas au Cameroun, où l'usine de Mbandjock de la SOSUCAM (Société Sucrière du Cameroun) a vu sa production multipliée par 4 entre 1965 et 2000, grâce à une augmentation de sa richesse usine, qui est passée au-dessus de la barre des $13 \%$, depuis 1986 . Le rendement de l'usine a franchit la barre des $10 \%$ pendant que les pertes totales sont au-dessous de la barre des $3 \%$ en 1991. Malgré l'allongement de la durée de la campagne depuis 1998, ces performances sont maintenues grâce à des investissements réguliers en matériels et des efforts par la maîtrise de la technique (Tedga et al., 2001). De même, Nadia et Mahmond (2006) ont affirmé que le rendement d'une raffinerie de canne dépend à la fois de la qualité technologique de la canne et de la qualité du processus technologique. De nombreux travaux ont été effectués sur l'économie d'énergie en sucrerie, mais très peu ont été consacrés à l'épuration et à la cristallisation du sucre (Elemva et ai., 2001).

Le présent travail analyse l'effet de la richesse de la canne à sucre, de l'extraction réelle et de la récupération du jus mélangé sur le rendement de l'usine et la récupération générale de la raffinerie de canne à sucre de la SUCAF-Cl Ferké 1, au cours des campagnes sucrières successives de 2003 à 2006.

\section{MATERIEL ET METHODES}

\section{MATERIEL}

Le matériel végétal utilisé à Ferké 1 se compose de 11 variétés commerciales de canne à sucre (Saccharum officinarum $L$.) réparties en variétés de début (NCO 376 et CO 449), de milieu (R570, Q75, R70367) et de fin de campagne (FR8069, M3145, C0957, C062175, SP701406 et SP701003). Les plantations villageoises représentent 5 à $8 \%$ du total des cannes produites (Pene et Kehe, 2005 ; Baudouin, 2001).

\section{MATERIELBIOLOGIQUE}

Le matériel biologique se compose des produits de l'usine répartis selon les différentes étapes du processus de transformation de la canne à sucre. Lors de l'extraction, les produits biologiques sont les jus des moulins et la bagasse. En ce qui concerne l'épuration, les produits biologiques sont le jus chaulé, les écumes et le jus clair. Au niveau de l'évaporation, le produit biologique est le sirop, tandis qu'au niveau de la cuite, ceux-ci sont représentés par la mélasse, les masses cuites et leurs égouts, ainsi que le sucre raffiné. 


\section{MATERIEL TECHNIQUE}

Il s'agit du matériel de technologie sucrière utilisé pour la production industrielle du sucre et le matériel de laboratoire utilisé pour le contrôle chimique. Celui-ci comprend plusieurs appareils, dont le polarimètre (ou saccharomètre) et le réfractomètre pour la mesure de la richesse en sucre (pouvoir rotatoire) et du brix (taux de matière sèche dissoute). Le matériel de technologie sucrière permet l'extraction et le raffinage du sucre extrait de la canne à sucre. Les équipements de production du sucre raffiné sont les moulins, les corps évaporateurs, les malaxeurs et les centrifugeuses.

\section{METHODES}

\section{Milieu d'étude}

L'étude a porté sur le complexe sucrier de Ferké 1 de la SUCAF-CI. II est situé dans le département de Ferkessédougou, à $17 \mathrm{Km}$ au Sud-Ouest de la ville. Ferké 1 est limité au Nord par la ville de Ferkessédougou, au Sud par la rivière Waha, à l'Est par la voie routière FerkéAbidjan et à l'Ouest par le fleuve BandamaBlanc. Ferké 1 dispose d'une superficie de 6000 ha de plantations industrielles sous irrigation et d'une usine ayant une capacité de broyage de 3500 t de cannes par jour, avec une production moyenne annuelle de 50000 t de sucre. L'usine produit du sucre roux en poudre, du sucre blanc en poudre et en morceaux. Le climat de la région de Ferkessédougou est du type tropical pré désertique ou Soudanien. La campagne de récolte s'étend de novembre à mars-avril, période qui correspond à la saison sèche, où la maturation (accumulation des réserves saccharifères) des cannes et la circulation des engins de récolte (ramassage et transport des cannes) sont plus aisées (Péné et Tuo, 1996).

\section{Récolte de la canne à sucre}

La veille, au soir, la portion de champ qui sera récoltée le lendemain est délimitée par des parefeux coupés à la main. Quand le vent cesse ou souffle faiblement, le feu est mis aux cannes et celui-ci s'éteint dès que les parties sèches (feuilles) aient été consumées. Le lendemain matin, dans le champ, le coupeur entre, sectionne la tige au ras du sol et l'étête à l'aide d'une machette. Ensuite, il retourne la tige et, avec la partie non tranchante de son outil, il enlève les feuilles en les prenant à rebrousse poil. Les tiges récoltées sont endainées à plat sur le sol, sur une zone préalablement débarrassée de feuilles et de déchets. Les ramasseuses se déplacent d'un tas de cannes à l'autre en prenant une pincée de cannes endainées qu'elles mettent dans la remorque roulant à leurs côtés. Les cannes sont ensuite transportées dans la remorque jusqu'à l'usine. Les variétés de début de campagne sont récoltées en novembre, celles du milieu de campagne à partir d'avril, tandis que les variétés de fin de campagne le sont au mois de mai.

\section{Processus de production du sucre raffiné}

Les chargements de cannes, qui arrivent par camions ou remorques à l'usine sont pesés et les cannes déchargées et stockées dans la cour de l'usine. Selon le rythme de fonctionnement de l'usine, un grappin fixé sur un portique dépose les cannes sur la table d'alimentation. Celles-ci sont lavées, puis défibrées par des coupecannes, avant de passer dans une série de 4 moulins (Hugot, 1987). Par imbibition composée, le saccharose est ainsi progressivement extrait. Le jus de canne est récupéré sous chaque moulin jusqu'au dernier, où il ne reste que le résidu fibreux ou bagasse (Alfa Azarté, 2005). Le jus est réchauffé à $105^{\circ}$, chaulé (pour permettre la précipitation des «impuretés») et sulfité (pour une meilleure élimination des composés azotés). Il est ensuite versé dans une cuve, qui permet de séparer, par décantation, le jus clair des écumes de défécation. Ces dernières, couramment appelées «boues» sont ensuite envoyées sur un tambour de filtration, où l'on extrait d'un côté du jus, qui sera chaulé et, de l'autre, des tourteaux riches en matières organiques (Hugot, 1987). Après décantation, on obtient un jus clair qui est envoyé dans plusieurs évaporateurs en série (appelés effets) où il perd $75 \%$ de son eau et prend une teinte brunâtre du fait de l'ébullition. Le sirop sortant de la dernière ébullition a été ensuite cristallisé sous vide partiel. Les 3 cuissons successives (appelés jets) ont permis de recueillir un maximum de sucre. La masse cuite a été centrifugée et le sucre roux récupéré et séché. Lors de «l'empâtage», le sucre roux a été déversé dans un malaxeur et mélangé par brassage avec un sirop chaud légèrement sous-saturé pour 
favoriser la dissolution superficielle des cristaux. La partie impure de la couche superficielle des cristaux est dissoute pour obtenir la masse-cuite qui a été essorée avec clairçage pour obtenir un «sucre d'affinage» puis dissous dans de l'eau chaude (Alfa Azarté, 2005). Le sirop trouble formé a été alcalinisé par l'addition de lait de chaux. Les impuretés précipitées ont été séparées du sirop par filtration. Après décoloration sur un lit de charbon actif, les sirops de refonte ont été purifiés. Le jus limpide est alors prêt à cristalliser en un sucre de qualité en 4 ou 5 jets de cristallisation.

\section{Contrôle chimique}

Le contrôle chimique consiste à suivre l'évolution de la qualité technologique de la canne à sucre broyée, lors des phases de broyage et de concentration (Hugot, 1987) par prélèvement des différents produits biologiques pour analyse au laboratoire. Meade et Chen (1977).

\section{Paramètres mesurés}

- Paramètres de la qualité technologique de la canne

Richesse usine $=100 \times[($ Tonnes Bagasse $\mathrm{x}$ Pol\% Bagasse) + (Tonnes JM x Pol\% JM)] / TCB

- Paramètres de la qualité du procédé

Extraction réelle $=$ Tonnes $\mathrm{Pol}$ jus mélangé $\mathrm{x}$ 100 / Tonnes Pol Cannes

Rendement Usine $=100 \times$ Tonne sucre extrait/ TCB

Gain de pureté pendant l'épuration (GPEP) = 100 x (pureté jus clair - pureté jus mélangé)/ pureté jus mélangé

Chute de pureté pendant l'évaporation (CPEV) = pureté du jus clair - pureté du sirop

Epuisement de la masse cuite $C(E M M C)=100$ $\mathrm{x}$ (pureté MCC - pureté mélasse)/ pureté MCC

Avec,

Tonnes Cannes Broyées $(\mathrm{TCB})=$ Tonnes Cannes Pesées + Stock de canne de la veille Stock de canne du jour
Tonnes Pol Cannes = Tonnes Pol jus mélangé + Tonnes Pol bagasse

Tonnes Pol d'un produit = Tonnes produit x Pol\% du produit / 100

Tonnes bagasse $=\mathrm{TCB}+$ Tonnes eau d'imbibition - Tonnes jus mélangé

Pureté $=100 \times$ Pol $\% /$ Brix $\%$

\section{Analyse statistique des données}

Les paramètres étudiés (rendement et richesse usine, extraction réelle, gain de pureté pendant l'épuration, chute de pureté pendant l'évaporation, épuisement de la masse cuite C) ont fait l'objet d'une analyse de variance, avec le logiciel statistique STATISTICA 6 FR. Les moyennes ont été comparées à l'aide du test de Newman-Keuls, au seuil de $5 \%$. Les coefficients de corrélation ( $r$ ) entre les différents paramètres ont été calculés, à l'aide de l'analyse des corrélations. Le coefficient de détermination $\left(R^{2}\right)$ a permis de quantifier la part de variance d'un paramètre en fonction de l'autre. Au terme de ces 4 années d'expérimentation, chacune des campagnes sucrières a été divisée en 3 grandes périodes (début, milieu et fin) et numérotées de 1 à 12 . Le début, le milieu et la fin de la campagne ont correspondu aux périodes 1,2 et $3 ; 4,5$ et $6 ; 7,8$ et 9 et 10,11 et 12 pour les années 2003, 2004, 2005 et 2006, respectivement.

\section{RESULTATS}

\section{CORRELATION ENTRE LES PARAMETRES ETUDIES}

L'étude des corrélations a montré que le rendement de l'usine de Ferké 1 a été corrélé négativement avec la chute de pureté pendant l'évaporation $\left(R^{2}=12,96 \%\right)$ et positivement avec la richesse usine $\left(R^{2}=43,56 \%\right)$, l'extraction réelle $\left(R^{2}=1,2 \%\right)$, le gain de pureté pendant l'épuration ( $\left.R^{2}=5,3 \%\right)$, l'épuisement de la masse cuite $C\left(R^{2}=1,7 \%\right)$. Le gain de pureté pendant l'épuration a été corrélé positivement avec l'épuisement de la masse cuite $C\left(R^{2}=\right.$ 13,0 \%) (Tableau 1). 
Tableau 1 : Corrélation entre les paramètres étudiés.

Correlation between the studied parameters.

\begin{tabular}{|c|c|c|c|c|c|c|}
\hline & E.RELLE & GPE & CPEV & EMCC & Richesse & Rendement \\
\hline E.RELLE & 1,0 & $-0,49$ & $-0,87$ & 0,48 & 0,43 & 0,11 \\
\hline GPE & & 1,0 & 0,47 & 0,36 & 0,35 & 0,23 \\
\hline CPEV & & & 1,0 & $-0,20$ & 0,22 & 0,36 \\
\hline EMCC & & & & 1,0 & $.0,09$ & 0,14 \\
\hline Richesse & & & & & 1,0 & 0,66 \\
\hline
\end{tabular}

E. réelle : extraction réelle ; GPE : gain de pureté pendant l'épuration ; CPEV : chute de pureté pendant l'évaporation ; EMCC : épuisement de la masse cuite $C$

\section{PARAMETRES DE LAQUALITE DU PROCEDE}

\section{Rendement de l'usine}

Le rendement moyen usine à Ferké 1 a été de $9,6 \pm 2,2 \%$ et compris entre $8,4 \%$ (période 3 ) et $10,4 \%$ (périodes 10 et 6 ) (Tableau 2). Le rendement usine a varié très peu entre 2003 et 2006. Les rendements usine des 12 périodes ont formé deux grands groupes : le $1^{\text {er }}$ groupe comprend les périodes $3,4,7,12,9$ et 8 , avec un rendement usine compris entre $8,4 \%$ et $9,7 \%$. Le second groupe comprend les périodes $5,11,10$ et 6 , avec un rendement usine compris entre $10,2 \%$ et $10,4 \%$ (Figure 1 ).

\section{Extraction réelle}

L'extraction réelle a été en moyenne de 90,6 \pm $2,4 \%$ et comprise entre $86,9 \%$ (période 3 ) et $92,7 \%$ (période 7 ) (Tableau 2). On peut scinder les 12 périodes en 3 grands groupes en fonction de leur extraction réelle. Les périodes $7,8,9$, 10 , 9 et 11 ont pour extractions réelles respectives $92,1,92,4,92,2,92,8$ et $91,6 \%$. Ces extractions réelles ont été les plus élevées du début de la campagne 2005 au milieu de la campagne 2006. Les périodes 1,2 et 3 (début, milieu et fin de la campagne 2003), possèdent les extractions réelles moyennes les plus faibles (respectivement de $88,4,87,9$ et $86,9 \%$ ), elles n'ont pas été significativement différentes. Les extractions réelles intermédiaires ont été obtenues lors des périodes 4 (début 2004 : 89,7\%), 5 (milieu 2004 : 90,5\%), 6 (fin 2004 : $90,5 \%$ ) et 12 (fin $2006: 90,5 \%$ ). L'extraction réelle de la période 4 n'a pas été significativement différente de celle des autres périodes de ce groupe. Dans l'ensemble, on a observé une augmentation de l'extraction réelle de 2003 à 2006 (Figure 2) .

\section{Gain de pureté pendant l'épuration}

Le gain de pureté pendant l'épuration a été en moyenne de $1,0 \pm 0,5 \%$ et compris entre $0,6 \%$ (période 10) et 1,4\% (périodes 6 et 5 ) (Tableau 2). On peut aussi subdiviser les 12 périodes en deux grands groupes en fonction du gain de pureté pendant l'épuration. Le 1er groupe comprend les périodes dont le gain de pureté pendant l'épuration a été inférieur à 1,0 ; il s'agit des périodes $9,10,8,1$ et 11 . Le second groupe comprenant les périodes $7,12,2,3,5,4$ et 6 et ont été caractérisées par un gain de pureté pendant l'épuration compris entre $1,0 \%$ et $1,4 \%$. Notons également que la période 1 a été caractérisée par un gain de pureté faible pendant l'épuration et appartient donc au 1er groupe (Figure 3). 
Tableau 2 : Données d'analyses statistiques élémentaires sur des paramètres étudiées. Elementary statistics data on the studied parameters.

\begin{tabular}{llcccccc}
\hline \multirow{2}{*}{ Périodes } & \multicolumn{7}{c}{ Paramètres étudiés } \\
\cline { 2 - 7 } & $\begin{array}{l}\text { E. réelle } \\
(\%)\end{array}$ & $\begin{array}{c}\text { GPE } \\
(\%)\end{array}$ & $\begin{array}{c}\text { CPEV } \\
\text { (point) }\end{array}$ & $\begin{array}{c}\text { EMCC } \\
(\%)\end{array}$ & $\begin{array}{c}\text { Richesse } \\
(\%)\end{array}$ & $\begin{array}{c}\text { Rendement } \\
(\%)\end{array}$ \\
\hline \multirow{4}{*}{ Début } & 1 & $88,5 \mathrm{~d}$ & $0,8 \mathrm{abc}$ & $1,4 \mathrm{f}$ & 21,8 & $13,4 \mathrm{abd}$ & $9,2 \mathrm{ab}$ \\
& 4 & $90,4 \mathrm{e}$ & $1,3 \mathrm{f}$ & $0,7 \mathrm{ab}$ & $24 ; 2$ & $12,3 \mathrm{abc}$ & $8,5 \mathrm{~b}$ \\
& 7 & $92,0 \mathrm{ab}$ & $1,0 \mathrm{f}$ & $0,9 \mathrm{e}$ & 24,3 & $11,7 \mathrm{c}$ & $9,2 \mathrm{ab}$ \\
& 10 & $92,7 \mathrm{~b}$ & $0,6 \mathrm{bc}$ & $0,6 \mathrm{abe}$ & 24,7 & $13,1 \mathrm{abcd}$ & $10,4 \mathrm{a}$ \\
& 2 & $87,9 \mathrm{~d}$ & $1,1 \mathrm{de}$ & $1,6 \mathrm{f}$ & 23,6 & $14,4 \mathrm{~d}$ & $9,7 \mathrm{ab}$ \\
Milieu & 5 & $90,1 \mathrm{ce}$ & $1,4 \mathrm{f}$ & $0 ; 5 \mathrm{ab}$ & 25,2 & $13,5 \mathrm{abd}$ & $10,2 \mathrm{a}$ \\
& 8 & $92,4 \mathrm{ab}$ & $0,8 \mathrm{abc}$ & $0,3 \mathrm{ac}$ & 23,3 & $12,6 \mathrm{abc}$ & $9,7 \mathrm{ab}$ \\
& 11 & $91,6 \mathrm{a}$ & $0,9 \mathrm{acd}$ & $0,3 \mathrm{ac}$ & 23,0 & $13,6 \mathrm{abd}$ & $10,3 \mathrm{a}$ \\
& 3 & $86,9 \mathrm{f}$ & $1,2 \mathrm{ef}$ & $1,6 \mathrm{f}$ & 24,4 & $12,5 \mathrm{abc}$ & $8,4 \mathrm{~b}$ \\
Fin & 6 & $90,5 \mathrm{c}$ & $1,4 \mathrm{f}$ & $0,7 \mathrm{ab}$ & 25,4 & $13,8 \mathrm{bd}$ & $10,4 \mathrm{a}$ \\
& 9 & $92,2 \mathrm{ab}$ & $0,6 \mathrm{~b}$ & $0,2 \mathrm{c}$ & 22,7 & $12,1 \mathrm{ac}$ & $9,3 \mathrm{ab}$ \\
& 12 & $90,4 \mathrm{c}$ & $0,9 \mathrm{aef}$ & $0,8 \mathrm{bd}$ & 22,4 & $12,1 \mathrm{abd}$ & $9,2 \mathrm{ab}$ \\
\hline Moyenne & & 90,6 & 1,0 & 0,8 & 23,6 & 12,9 & 9,6 \\
\hline Ecart type & & $2,4 *$ & $0,5 *$ & $0,8 *$ & $5,2 \mathrm{~ns}$ & $2,4 *$ & $2,2 *$ \\
\hline
\end{tabular}

E. réelle : extraction réelle ; GPE : gain de pureté pendant l'épuration ; CPEV : chute de pureté pendant l'évaporation ; EMCC : épuisement de la masse cuite $\mathrm{C} ;$ ns : différence non significative ${ }^{*}$ différence significative ; a, b, c... : les moyennes suivies de la même lettre ne diffèrent pas significativement, au seuil de $5 \%$.

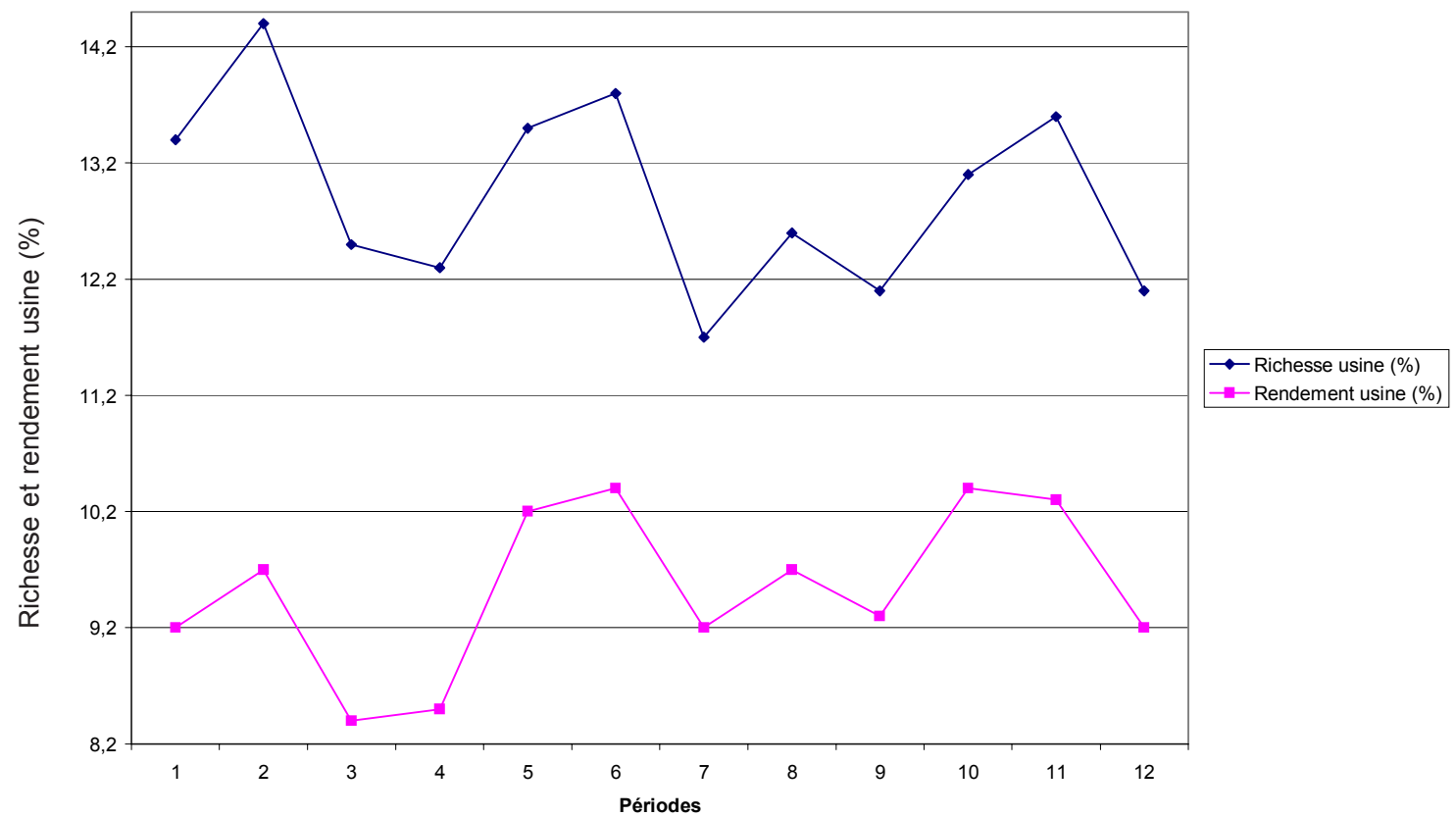

Figure 1 : Evolution du rendement et de la richesse usine en fonction du temps à Ferké 1.

Evolution of yield and factory richness, as a function of time at Ferké 1. 


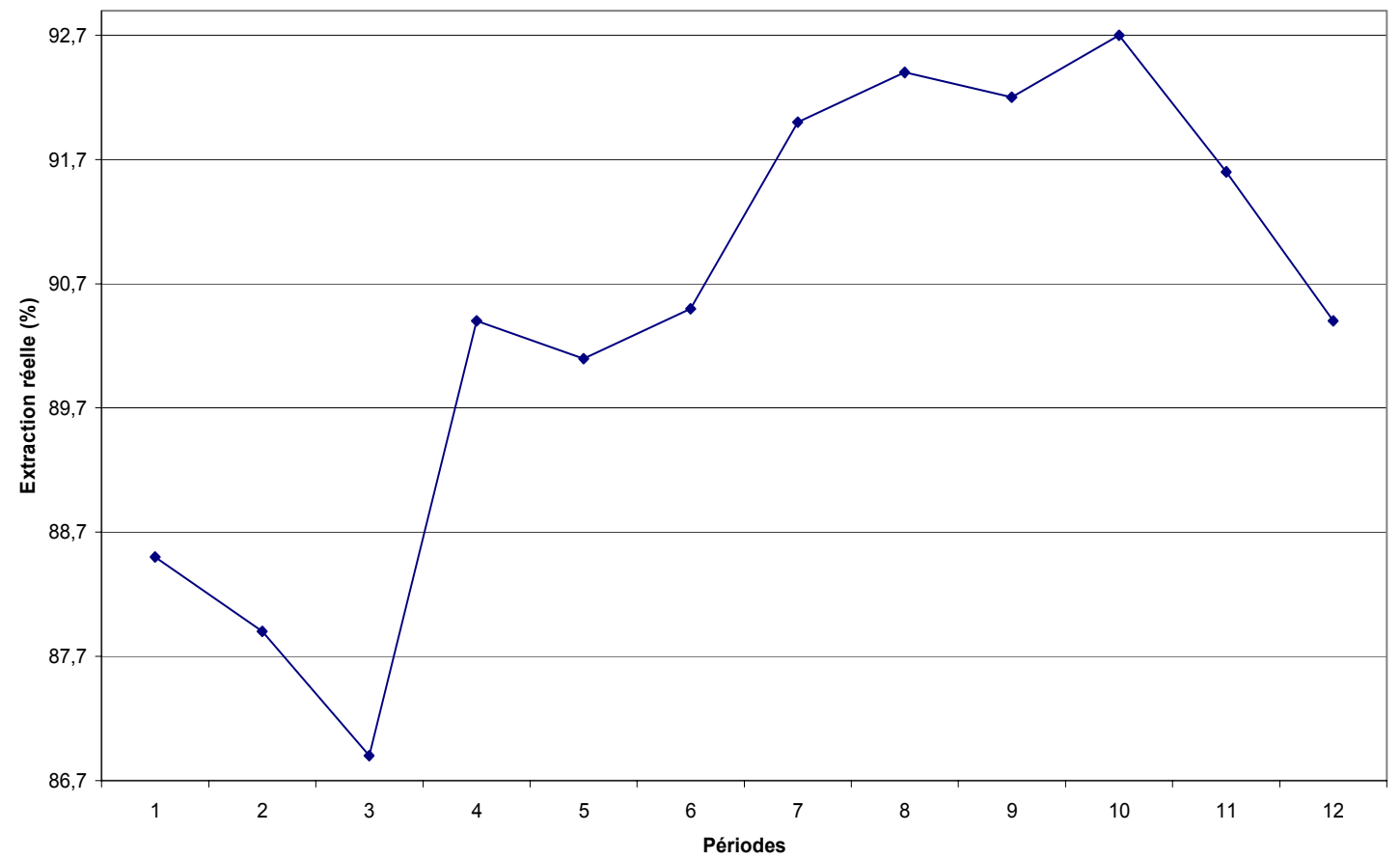

Figure 2 : Evolution de l'extraction réelle de l'usine de Ferké 1 de 2003 à 2006.

Evolution of real factory extraction at the Ferke 1 plant, from 2003 to 2006.

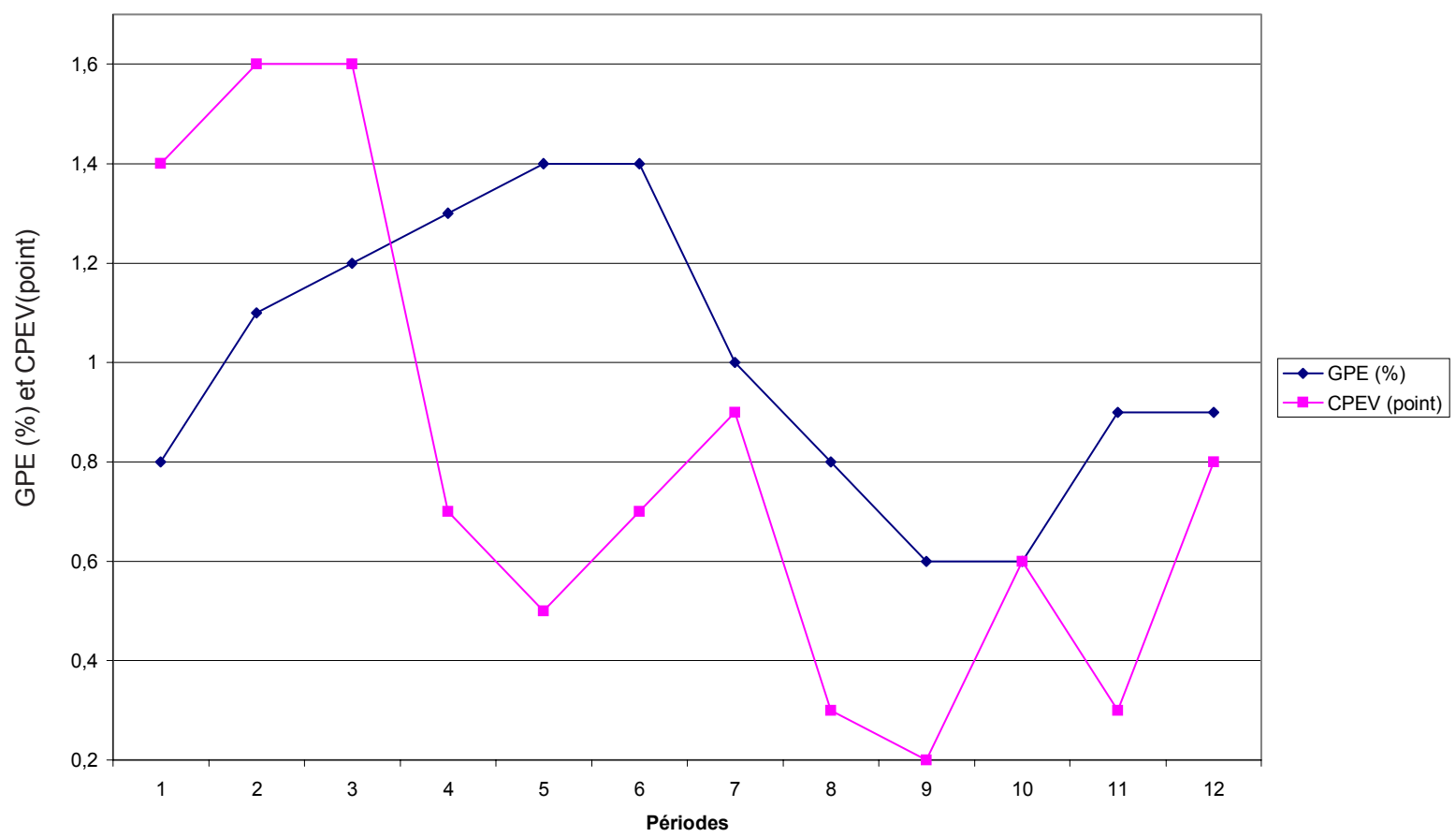

Figure 3 : Evolution du gain de pureté pendant l'épuration (GPE) et de la chute de pureté pendant l'évaporation (CPEV) de l'usine de Ferké 1.

Evolution of the gain of purity during the epuration and the falling of purity during the evaporation of the Ferke 1 sugar plant extraction. 


\section{Chute de pureté pendant l'évaporation}

La pureté du jus a baissé pendant l'évaporation en moyenne de $0,8 \pm 0,8$ point et a été comprise entre 0,2 (période 9 ) et 1,6 (périodes 2 et 3 ) (Tableau 2). On peut aussi scinder les 12 périodes en deux grands groupes en fonction de la chute de pureté pendant l'évaporation : le 1 er groupe comprend les périodes dont la chute de pureté pendant l'évaporation a été inférieure à 1,0 point ; il s'agit des périodes $9,11,8,6,5$, $10,4,12$ et 7 . Le second groupe a eu les chutes de pureté pendant l'évaporation les plus élevées (comprises entre 1,3 points et 1,4 points) et a été caractérisé par les périodes 1,2 et 3. Dans l'ensemble, la chute de pureté pendant l'évaporation a baissé de 2003 à 2006 (Figure 3). Notons que la période 1 a été caractérisée par des chutes de pureté pendant l'évaporation les plus importantes.

\section{Epuisement de la masse cuite C}

L'épuisement de la masse cuite $C$ a été en moyenne de $23,6 \pm 5,2 \%$ et compris entre 21,8 (période 1) et 25,4 (période 6) (Tableau 2). Ce paramètre n'a pas varié significativement de 2003 à 2006. Notons néanmoins que la valeur moyenne de l'épuisement de la masse cuite C la plus faible a été obtenue au cours de la période 1.

PARAMETRES DE QUALITE DE LA CANNE A SUCRE

\section{Richesse de l'usine}

La richesse de l'usine moyenne a été de $12,9 \% \pm 2,4$ et comprise entre $11,7 \%$ (période 7 ) et $14,4 \%$ (période 2) (Tableau 2). Aucune richesse usine n'a été significativement différente des autres. On peut regrouper les 12 périodes en deux grands ensembles en fonction de leur richesse usine. La 1 ère grande période comprend les périodes $7,12,9,4,3$ et 8 , avec une richesse usine comprise entre 11,8 et $12,6 \%$. Ces périodes sont caractérisées également par un rendement usine faible (compris entre 8,4 et $9,7 \%$ ). La seconde grande période comprend les périodes $10,1,11,5,6$ et 2 , avec un rendement usine compris entre 13,1 et $14,4 \%$. En dehors de la période 1, toutes les autres périodes $(10,11,5,6$ et 2$)$ ont été caractérisée par un rendement usine élevé (compris entre 10,2 et 10,4\%).

\section{DISCUSSION}

Le rendement de l'usine de Mbandjock de la SOSUCAM a augmenté de 1,58 points de 1965 à 2000, pour atteindre 9,59 \% (Tedga et al., 2001). Fauconnier et Bassereau (1979) ont estimé que le rendement d'une raffinerie de canne peut être compris entre 8 et $14 \%$. Selon Alfa Azarte (2005), la canne à sucre peut contenir jusqu'à $16 \%$ de saccharose au niveau des tiges. La richesse de l'usine de Mbandjock a varié entre 10,32 et $12,48 \%$ de 1965 à 2000 (Tedga et al., 2001).

Le rendement usine a été corrélé positivement avec l'extraction réelle, le taux d'épuisement de la masse cuite $C$, le gain de pureté pendant l'épuration et la richesse de l'usine. Il a été par contre, corrélé négativement avec la chute de pureté pendant l'évaporation. Des conclusions similaires ont été obtenues par Thangavelu (2004). Le faible rendement usine obtenu lors de la période 1, malgré une richesse usine des plus élevées, serait donc dû à un faible gain de pureté pendant l'épuration, à un faible épuisement de la masse cuite $C$ et à une forte chute de pureté pendant l'évaporation enregistrés au cours de cette période.

Selon Hugot (1987), l'extraction réelle normale d'une batterie de moulins doit être comprise entre 94 et $95 \%$ tandis que pour Amrani (2006), les moulins peuvent permettre d'extraire 92 à $96 \%$ du saccharose contenu dans la canne à sucre. L'extraction réelle de l'usine de Ferké 1 $(90,6 \%)$ n'a donc pas été assez élevée. En Inde, Thangavelu (2004) a montré que le pourcentage d'extraction du jus a varié avec la variété et l'état de maturité de la canne dans une étude sur le taux d'extraction de 30 clones de canne à sucre (13 cultivars de maturation précoce et 17 de maturation tardive), à des étapes de récolte différentes $(8,9,10,11,12$ et 13 mois). Le pourcentage d'extraction du jus par clone a varié entre $49,89 \%$ pour la variété Co 7204 et $60,96 \%$ pour la variété Co 419 .

A Kohala à Hawaii, l'écart de pureté entre le jus clair et le jus mélangé a varié entre - 0,3 et + 0,1\% (Hugot, 1987). La firme américaine Dorr, fabriquant de clarificateurs, a mis au point un procédé de défécation appellé clarification composée qui exige la séparation du jus en deux parties : le jus primaire, ou vesou de pression sèche, fourni par le défibreur et/ou le $1^{\text {er }}$ moulin, le jus secondaire, formé par les jus de pression 
humide. La clarification composée consiste à traiter séparément ces deux catégories de jus, qui présentent entre elles des différences significatives. Dans une expérience faite à Kohala à Hawaii, à l'aide de la clarification composée, le gain de pureté obtenu a été de + 1,5 à $+1,9 \%$ (Hugot, 1987). En Inde, Balakrishnan et al. (2001) ont obtenu un gain de pureté pendant l'épuration par la chaux de 0,5 à 1 point. L'utilisation de la chaux pendant l'épuration a permis de neutraliser les acides organiques contenus dans le jus de canne (Chen, 2000) et de séparer les mousses ou écumes riches en sels de calcium insolubles, l'albumine coagulée, les graisses, les cires, ainsi que les fines bagasses (Amrani, 2006). Le chaulage permet une amélioration de la couleur du jus de canne et une baisse de concentration en sucres invertis, en dextranes et en oligosaccharides (Eggleston, 2002). L'augmentation de la pureté du jus mélangé au jus clair est donc due à l'élimination des impuretés du jus de canne capable de gêner la cristallisation soit en retenant le saccharose (par des liaisons hydrophiles, hydrophobes, etc.), diminuant ainsi le rendement de cristallisation, soit en s'incrustant au cristal du sucre (polysaccharides, colorants, etc.), réduisant ainsi sa pureté (Decloux et al., 1999).

A Java, Hugot (1987) a constaté une élévation de la pureté du jus pendant l'évaporation égale à 0,8 , pour les sucreries à défécation, 0,9 pour les sucreries à sulfitation et 0,4 pour les sucreries à carbonatation. Toujours selon le même auteur, lorsque le jus est porté à une haute température pendant l'évaporation, le sucre qu'il contient subit une décomposition par inversion (hydrolyse) d'autant plus importante que la température et l'acidité sont plus élevées. Cette hydrolyse est provoquée par la chaleur appliquée aux corps évaporateurs (Amrani, 2006). Cela se traduit par une chute du $\mathrm{pH}$ et de la pureté entre le jus clair et le sirop, entraînant une perte de sucre. La baisse de pureté du sirop peut aussi s'expliquer par l'encrassement des corps évaporateurs, ce qui induit une haute température pour l'évaporation avec présence d'une quantité de chaux n'ayant pas été éliminée lors de la clarification et augmentation de la quantité de matières sèches (Hugot, 1987).

Le taux d'épuisement de la masse cuite $\mathrm{C}$ a été inférieur à celui de $56,6 \%$, valeur recommandée par Hugot (1987) qui affirme que c'est l'épuisement de la masse cuite $C$ qui est de loin le plus important, parce qu'il commande la pureté de la mélasse et, par conséquent, la récupération du sucre. Kapseu et al. (1993) ont montré que, lors de la production du sucre, les étapes qui entrainent le plus de pertes en sucre sont, par ordre décroissant, la raffinerie, le jet A, l'épuration, le jet $C$ et l'évaporation.

La richesse de la canne à sucre a été le principal facteur qui a affecté le rendement de la raffinerie de Ferké 1 de 2003 à 2006. L'usine de Ferké 1 peut donc encore accroître son rendement puisque son extraction réelle et son taux d'épuisement de la masse cuite $C$ ont été inférieurs aux seuils de $94 \%$ et 56,6\% respectivement (Hugot, 1987). Sa richesse usine peut encore être améliorée en prenant toutes les dispositions pour réduire les pertes en saccharose entre la plantation, l'usine et au cours du processus de fabrication du sucre, notamment au broyage et à l'évaporation. Pour obtenir le rendement le plus élevé possible, il faut donc livrer à la raffinerie la canne la plus riche possible en saccharose. La meilleure canne à sucre se décline conjointement sous deux formes : celle atteignant une certaine maturité (11 à 12 mois) et celle pour laquelle la concentration en saccharose est maximale. La plupart des sucreries donnent des ordres de coupe basés sur l'âge de la canne. Ce n'est pas une méthode scientifique puisque la maturité est influencée par la période de planting, les pratiques de la récolte, les conditions climatiques, etc... Une canne peut être considérée comme apte à la récolte si elle a atteint un minimum de $16 \%$ de saccharose et $85 \%$ de pureté. La récolte de la canne à sucre au bon moment, c'est à dire au maximum de maturité, en adoptant de bonnes techniques, est nécessaire pour réaliser le poids maximal de canne usinable, avec un minimum de pertes au champ. D'un autre côté, une récolte de la canne avant ou après le pic de maturité, avec des méthodes de récolte impropres, conduit à des pertes de rendement en canne, en récupération du saccharose, et conduit à un jus de canne de mauvaise qualité et à des problèmes lors $\mathrm{du}$ broyage dus aux matières étrangères (feuilles, racines, bouts blancs, etc.). Au champ, il faut couper la canne au niveau du sol pour que le sucre qui se trouve dans les entrenoeuds les plus bas et les plus riches en sucre soit récupéré pour accroître le rendement en canne et la quantité de sucre. II faut aussi couper le sommet de la canne à la bonne hauteur afin d'éliminer les entrenoeuds immatures. II faut également 
bien nettoyer la canne en éliminant les matières étrangères et acheminer le plus rapidement possible la canne récoltée à l'usine pour broyage (Chen., 2000).

La seule mesure pratique pour minimiser l'inversion et la transformation du saccharose est de réduire au maximum le délai entre la coupe et le broyage de la canne (Chen, 1977). En Australie, la plupart des sucreries cherchent à faire en sorte que le temps qui s'écoule entre la récolte et le broyage à l'usine ne dépasse pas $15 \mathrm{~h}$, alors que la durée moyenne est approximativement de $9 \mathrm{~h}$. Le gain d'efficacité, à travers une meilleure intégration des secteurs de la récolte et du transport a été considéré comme de la plus haute priorité dans ce pays (Hildebrand, 2002). Higgins et lan (2005) ont crée un système de récolte et de transport qui a permis d'accroître les gains financier dans l'industrie sucrière australienne. Cette étude a permis à la région de passer de la récolte au cours de la journée à une récolte qui dure $18 \mathrm{~h}$ en moyenne pendant la campagne de 2003. A la Réunion, comme à l'île Maurice, un modèle d'approvi-sionnement des sucreries de canne, appelé MAGI (Modèle d'Approvisionnement Global informatisé), a été élaboré afin d'évaluer et de comparer différents scénarios d'organisation des approvisionnements à l'échelle des bassins de collecte. MAGI se compose en 3 modules : un module de planification qui définit un plan hebdomadaire de livraisons de cannes des unités de production à l'usine en passant par les transporteurs ou centres de réception, un module de simulation qui met en œuvre le plan sur la durée de campagne, un module de transformation qui évalue la quantité de sucre produite par semaine, à partir des résultats des 2 modules précédents. L'objectif de MAGI est de définir des voies d'évolution envisageables pour les industries et les planteurs, afin de réduire les coûts de production et d'exploiter, au mieux, I'hétérogénéité des bassins canniers (Lejars et al., 2004).

\section{CONCLUSION}

Cette étude a montré que, de la campagne 2003 à la campagne 2006, le rendement de la raffinerie de canne de Ferké 1 a été affecté principalement par la richesse de la canne à sucre, mais aussi, par la qualité du processus de production du sucre granulé. L'extraction réelle, le rendement et la richesse de l'usine, ainsi que le gain de pureté pendant l'épuration, la chute de pureté pendant l'évaporation et l'épuisement de la masse cuite $C$ ont été respectivement de 90,6 ; 9,$6 ; 12,9 ; 1,0 ; 0,8$ point et $23,6 \%$. Hormis le rendement de l'usine qui a varié légèrment et l'épuisement de la masse cuite $\mathrm{C}$ qui n'est pas resté constant, tous les autres paramètres étudiés ont connu une variation significative entre 2003 et 2006 . L'amélioration du rendement de l'usine de Ferké 1 passe donc principalement par la réduction du délai entre la coupe et le broyage de la canne à sucre, afin de lutter contre la détérioration de la canne, car tous les sous produits de cette détérioration réduisent l'efficience des différentes étapes du processus de production du sucre et la qualité du sucre produit. II faut aussi procéder à la formation continue du personnel responsable de la fabrication du sucre, à l'entretien du matériel existant, à l'acquisition de nouveau matériel de technologie sucrière et enfin, il convient de mettre en place un programme de sélection variétal qui permettra, à long terme, de disposer d'un matériel végétal à haut rendement, performant au plan de ses qualités technologiques et qui résiste à la détérioration postrécolte.

\section{REFERENCES}

Anonyme. http://www.bnetd.ci/ (Page consultée le 29 avril 2010)

Arzate A. 2005. Extraction et raffinage du sucre de canne. Centre de recherche, de développement et de transfert technologique en acériculture (ACER), SaintNorbert d'Arthabaska. $44 \mathrm{p}$.

Amrani M. 2006. Détermination de la qualité du pain de sucre à partir des concentrations des sucres réducteurs. AJST, 7(1): 1 - 7.

Andrew H. and I. Davies. 2005. A simulation model for capacity planning in sugarcane transport. Computers and Electronics in Agriculture. 47(2) : 85 - 102.

Balakrihnan M., Dua M. and P. N. Khaimar. 2001. Significance of membrane Type and Feed Stream in the Ultrafiltration of Sugarcane juice. Separation Science and Technology, $36(4)$ : 619 - 637.

Baudouin M. 2001. L'industrie sucrière en Afrique Francophone. AFCAS/ GPS - $3^{e}$ rencontre en langue française sur la canne à sucre. p 31 - 40. 
Chen C. P. 2000. Manual del azúcar de caña. Edicion Limusa, Mexico. 32 - 37, 41 - 43, $163-190$.

Decloux M., Tatoud L. et A. Mersad. 1999. Rétention des impuretés de refontes de sucre roux de canne par filtration tangentielle. Association AVH - $6^{\text {e }}$ Symposium - Reims.

Eggleston G. 2002. Deterioration of cane juice sources and indicators. Food Chemistry. 78 (1) : 95 - 103.

Elemva A., Tedga N., Kapseu C. et J. P. Champeaux. 2001. Optimisation de la sucrerie raffinerie de Mbandjock au Cameroun. AFCAS/ GPC. $3^{\mathrm{e}}$ rencontre en Langue Française sur la canne à sucre. p 194 - 201.

Fauconnier R. et D. Bassereau. 1979. La canne à sucre. Maisonneuve \& Larose, Paris. $563 \mathrm{p}$.

Hildebrand C. 2002. An independent assessment of the sugar industry. Report to the Hon Truss MP, Minister for Agriculture, Fisheries and Forestry, Australia.

Hugot E. 1987. La sucrerie de canne ; PARIS, éd. DUNOD. 1018 p.

Kapseu C., Ali Ahmed et N. Tedg. 1993. Contribution à la reduction des pertes à la sucrerie. Journal of food engineering. $20(1): 45-53$.

Lejars C., Auzoux S. et P. Y. Le Gal. 2004. In/ La canne une passion à partager : rencontres internationales pluridisciplinaires octobre 2002. [Cd- Rom]. Piton Saint-Lieu : Seml Réunion Museo. Colloque sur les pers- pectives de développement de la canne à sucre en milieu insulaire, 2002 - 10 - 02/ 2002-10-05, Stella Matutina, Réunion. 10p

Mead G. P. and J. C. P. Chen. 1977. Meade-Chen Cane Sugar Handbook. New York. John Wiley and Sons. $10^{\text {th }}$ edition ; $845 \mathrm{p}$.

Nadia M. A. and M. A. Khwaja. 2006. Study on effluents from selected sugar mills in Pakistan: Potential Environmental, Health, and Economic Consequences of an Excessive Pollution Load. Sustainable Development Policy Institute (SDPI). Islamabad, Pakistan. 42 p.

Péné C. B. et K. Tuo. 1996. Corrélation eaurendement en culture de canne à sucre : cas du complexe sucrier de Ferké 2 (Côte d'Ivoire). Agronomie Africaine VIII (2) : $97-111$.

Pene C. B. et M. Kehe. 2005. Performances de trois variétés de canne à sucre soumises au rationnement hydrique en prématuration au nord de la Côte d'Ivoire. Agronomie Africaine 17 (1): 7 - 18.

Tedga N., Kapseu C., Elemva A., et J. P. Champeaux. 2001. Amélioration des paramètres critiques en sucrerie de canne (Mbandjock, Cameroun). AFCAS/ GPC. $3^{\mathrm{e}}$ rencontre en Langue Française sur la canne à sucre. p 186 - 193.

Thanggavelus S. 2004. Juice extraction percent in sugarcane clones an dits relationship with important yield and juice quality characteristics. Sugarcane Breeding Institute, Coimbratore 641007, Indian Sugar. 54 (4) : 269 - 274. 